\title{
Commentary on Photoacoustic Tomography
}

\author{
Ralph P. Mason \\ Department of Radiology, University of Texas Southwestern Medical Center, Dallas, Texas
}

\section{$\mathbf{S}$} of the United States, over the past $10 \mathrm{y}$ has greatly promoted noninvasive approaches to studying pathophysiology, tumor progression, and response to therapy as well as pharmacokinetics and biodistribution of potential therapeutics. Many institutional resource facilities are now equipped to provide routine in vivo optical imaging (bioluminescent and fluorescent imaging), CT, PET, SPECT, MR imaging, and ultrasound. Within the past $5 \mathrm{y}$, a new technology has become commercially available: photoacoustic tomography (PAT), the tool exploited by Bohndiek et al. to evaluate tumor blood vessel regression and normalization in response to the experimental antiangiogenic therapy trebananib, as reported in the current issue of The Journal of Nuclear Medicine (1).

Effective modern small-animal imaging resources offer a range of instrumentation. As context for the report using PAT, each modality has notable strengths and limitations. A common characteristic is noninvasiveness allowing repeated measurements with respect to acute interventions or facilitating long-term evaluation of chronic changes. General considerations for a given application include availability and access, cost, spatial and

\section{See page 1942}

temporal resolution, and depth of signal penetration and signal sensitivity. Optical imaging such as fluorescence provides a vast array of chromophores, offering versatile contrast agents and stains for microscopy. However, strong scattering by turbid medium and tissues severely limits the depth resolution. MR offers exquisite tissue contrast and high-resolution anatomy at essentially any depth (mouse to human) but is relatively expensive and slow and requires high concentrations of reporter molecules (typically millimolar range). Proton nuclear magnetic resonance can allow molecular identification, albeit with sophisticated water and fat-suppression requirements. Use of heteronuclei, which occur at naturally low concentrations, avoids background signal, allowing massive isotope enrichment and signal amplification (e.g., ${ }^{13} \mathrm{C}$ ), which may be enhanced further using dynamic hyperpolarization (2). Exogenous ${ }^{19} \mathrm{~F}$ also provides versatile agents with exquisite sensitivity to the microenvironment and a large

\footnotetext{
Received Sep. 1, 2015; revision accepted Sep. 2, 2015.

For correspondence or reprints contact: Ralph P. Mason, Department of Radiology, UT Southwestern Medical Center, 5323 Harry Hines Blvd., Dallas, TX 75390-9058.

E-mail: Ralph.Mason@UTSouthwestern.edu

Published online Sep. 17, 2015.

COPYRIGHT (c) 2015 by the Society of Nuclear Medicine and Molecular Imaging, Inc.

DOI: $10.2967 /$ jnumed.115.165183
}

chemical shift range so that multiple reporters may be assessed simultaneously (3). CT is essentially an anatomic imaging approach, with limited contrast agents, although multispectral CT is emerging. PET and SPECT offer high sensitivity, although limited spatial resolution, and are associated with radiation, necessitating logistic costs and complexity associated with radioactivity. Ultrasound provides spatial resolution at depth, although there are few contrast agents other than microbubbles.

Photoacoustic tomography is a multimodality technique using optical excitation and ultrasound detection. Excitation of a chromophore causes local heating, thermoelastic expansion generating mechanical waves, which may be detected using ultrasonic transducers. Ideal PAT contrast agents exploit high light absorption and are generally inefficiently fluorescent. They are generally chosen to be active in the optical window of $600-1,000 \mathrm{~nm}$. Notably, oxy- and deoxyhemoglobin are strong endogenous chromophores with unique spectra, allowing spectral unmixing and assessment of vascular oxygenation.

The photoacoustic effect was first described some $100 \mathrm{y}$ ago, but it is the recent availability of commercial small-animal imaging devices that promises the opportunity for widespread applications. Until recently, PAT was restricted to pioneering laboratories, such as those of Emelianov (4), Wang (5), Beard (6), and Ntziachristos (7), who had the engineering acumen and laboratories to design, build, and maintain devices. In the past $5 \mathrm{y}, 3$ manufacturers have established instruments allowing more general imaging scientists to exploit the technology.

Notably, VisualSonics (Fuji), iThera, and Endra manufacture instruments in a price range amenable to National Institutes of Health Shared Small Instrumentation grants. Each system uses somewhat different instrument architecture, but common characteristics include a laser with a range of wavelengths, typically 680-980 nm, and a standard resolution of 50-300 $\mu \mathrm{m}$, allowing the detection of small structures, notably arterioles. Data may be processed using independent software-based environments such as MATLAB, although increasingly unmixing, deconvolution and data presentation software is included with the instruments. The VisualSonics LAZR system is an add-on to the Vevo ultrasound system, whereas iThera MSOT and Endra Nexus are standalone systems. iThera specifically markets a range of instruments including various numbers of transducers, 64,128 , or 256 , providing a range of spatial coverage and is currently introducing an added ultrasound capability.

Much PAT has been devoted to evaluating hemoglobin and changes in oxy/deoxyhemoglobin ratios, because these are convenient endogenous materials. Melanin is another endogenous chromophore offering strong signal and necessitating different analysis algorithms for black mice. Melanin offers potential as a transgene reporter. Popular current reporter genes such as green 


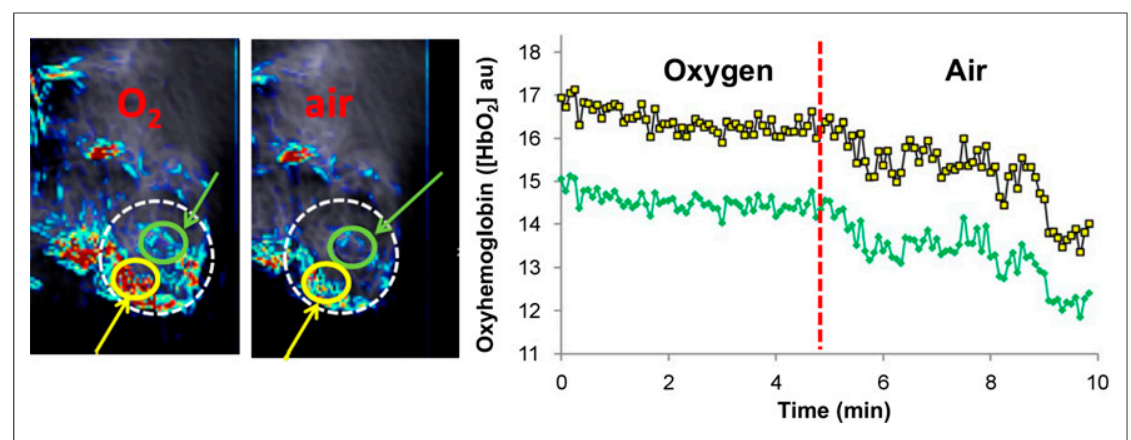

FIGURE 1. PAT of PC3 tumor with respect to gas challenge. Multiwavelength photoacoustic imaging was acquired using iThera MSOT 256TF device based on 5 wavelengths $(715,730,760,800$, and $850 \mathrm{~nm})$, signal unmixing, and backprojection reconstruction to achieve images of oxy- (shown) and deoxy- (not shown) hemoglobin. White circle outlines PC3 tumor growing subcutaneously in severe combined immunodeficiency mouse. Green and yellow circles show regions of interest with corresponding traces for temporal variations over $10 \mathrm{~min}$ at right. Anesthetized mouse initially breathed oxygen (with $1.5 \%$ isoflurane anesthesia) followed by air (red dotted line) after $5 \mathrm{~min}$.

fluorescent protein and mCherry are not effective for PAT contrast, but reports suggest that new longer-wavelength proteins such as near-infrared fluorescent protein hold promise $(8)$. Notable exogenous contrast agents include indocyanine green, methylene blue, and various nanoparticles, particularly gold (9).

In the study of Bohndiek et al., the authors probe oxy- and deoxyhemoglobin and total hemoglobin as an indication of tumor vascular development. Whole-mouse images were captured in $12 \mathrm{~s}$ at each of 3 wavelengths. Three-dimensional reconstruction provided $100 \times 100 \times 100 \mu \mathrm{m}$ voxels. Major blood vessels were identifiable, but within the tumor the capillary bed was below spatial resolution. As such, signal was assessed for the pertinent region of interest. This was not unlike blood oxygen level dependent MR imaging, in which tumor blood vessels are generally not identifiable within tumors, but a voxel signal response is exploited (10). The studies were conducted over several days, indicating chronic vascular normalization. As validation, indocyanine green was infused intravenously, and the signal provided a correlative confirmative indication of vascular extent. Data were also confirmed by immunohistochemistry and blood draws, revealing variations in specific protein correlations indicative of vascular development. Bohndiek et al. evaluated orthotopic ovarian tumors, emphasizing the depth resolution of the technique. Indeed, another popular site for PAT investigations has been orthotopic pancreatic tumors (11).

The work of Bohndiek et al. focused on longitudinal changes. PAT exhibits high temporal resolution, allowing the detection of rapid dynamics in response to acute interventions. Frequently, changes in oxy- and deoxyhemoglobin are reported with respect to an oxygen breathing challenge. For comparison, we provide an example of such an observation achieved shortly after acquiring our own commercial PAT system (Fig. 1). This emphasizes the ability to achieve PAT results without substantial historic experience in the modality. However, we are indebted to the advice of experienced investigators, notably Professors Emelianov (UT Austin) and McNally (University of Louisville) and Clinton Hupple, the applications engineer.
PAT is clearly not as easy to implement or interpret as optical imaging, but the 3-dimensional tissue definition is attractive. Currently, PAT is limited to mice and rats although results have been presented in humans at several centimeters' depth. In our experience, several criteria are crucial: tissue must be depilated; nude mice are ideal and easiest, though hair removal with agents such as NAIR (Church \& Dwight) can be highly effective (12). The coupling medium must be clean and bubble free: this may apply to the water bath and certainly to any coupling gel. Image focusing due to speed of sound should be straightforward but is an acquired skill.

PAT is at the exciting phase of a new technology becoming available to the wider scientific community. At this stage, validation experiments are generally provided, but increasingly the validity and robustness of PAT is accepted. Meanwhile, many new research applications of PAT are becoming feasible.

\section{DISCLOSURE}

Data for Figure 1 were kindly provided by James (Trey) Campbell and Dr. Li Liu in my laboratory with support from NIH P30 CA142543, S10 OD018094, and CPRIT RP140285. No other potential conflict of interest relevant to this article was reported.

\section{REFERENCES}

1. Bohndiek SE, Sasportas LS, Machtaler S, Jokerst JV, Hor, S, Gambhir SS. Photoacoustic tomography detects early vessel regression and normalization during ovarian tumor response to the antiangiogenic therapy trebananib. J Nucl Med. 2015;56:1942-1947.

2. Malloy CR, Merritt ME, Sherry AD. Could C-13 MRI assist clinical decisionmaking for patients with heart disease? NMR Biomed. 2011;24:973-979.

3. Yu J-X, Hallac RR, Chiguru S, Mason RP. New frontiers and developing applications in ${ }^{19}$ F NMR. Prog Nucl Magn Reson Spectrosc. 2013;70:25-49.

4. Bayer CL, Joshi PP, Emelianov SY. Photoacoustic imaging: a potential tool to detect early indicators of metastasis. Expert Rev Med Devices. 2013;10:125-134.

5. Wang LV, Hu S. Photoacoustic tomography: in vivo imaging from organelles to organs. Science. 2012;335:1458-1462.

6. Beard P. Biomedical photoacoustic imaging. Interface Focus. 2011;1:602-631.

7. Taruttis A, van Dam GM, Ntziachristos V. Mesoscopic and macroscopic optoacoustic imaging of cancer. Cancer Res. 2015;75:1548-1559.

8. Filonov GS, Piatkevich KD, Ting LM, Zhang JH, Kim K, Verkhusha VV. Bright and stable near-infrared fluorescent protein for in vivo imaging. Nat Biotechnol. 2011;29:757-761.

9. Poon W, Heinmiller A, Zhang X, Nadeau JL. Determination of biodistribution of ultrasmall, near-infrared emitting gold nanoparticles by photoacoustic and fluorescence imaging. J Biomed Opt. 2015;20:066007.

10. Zhao D, Pacheco-Torres J, Hallac RR, et al. Dynamic oxygen challenge evaluated by NMR $\mathrm{T}_{1}$ and $\mathrm{T}_{2} *$ : insights into tumor oxygenation. NMR Biomed. 2015;28:937-947.

11. Kimbrough C W, Khanal A, Zeiderman M, et al. Targeting acidity in pancreatic adenocarcinoma: multispectral optoacoustic tomography detects $\mathrm{pH}$-low insertion peptide probes in vivo. Clin. Cancer Res. June 29, 2015 [Epub ahead of print].

12. Kimbrough CW, Hudson S, Khanal A, Egger ME, McNally LR. Orthotopic pancreatic tumors detected by optoacoustic tomography using Syndecan-1. J Surg Res. 2015;193:246-254. 